\title{
André Torre et Jean-Eudes Beuret - Proximités territoriales, Construire la gouvernance des territoires, entre conventions, conflits et concertations - Paris, 2012, Economica Anthropos, 105 pages
}

\section{Maryline Filippi}

\section{(2) OpenEdition}

\section{Journals}

Édition électronique

URL : http://journals.openedition.org/economierurale/4141

DOI : $10.4000 /$ economierurale. 4141

ISSN : 2105-2581

Éditeur

Société Française d'Économie Rurale (SFER)

Édition imprimée

Date de publication : 15 novembre 2013

Pagination : 107-109

ISSN : 0013-0559

Référence électronique

Maryline Filippi, «André Torre et Jean-Eudes Beuret - Proximités territoriales, Construire la gouvernance des territoires, entre conventions, conflits et concertations - Paris, 2012, Economica Anthropos, 105 pages », Économie rurale [En ligne], 338 | novembre-décembre 2013, mis en ligne le 15 octobre 2013, consulté le 22 septembre 2020. URL : http://journals.openedition.org/economierurale/ 4141 ; DOI : https://doi.org/10.4000/economierurale.4141 
André TORRE et Jean-Eudes BEURET

\section{Proximités territoriales : construire la gouvernance des territoires, entre conventions, conflits et concertations}

Paris, Economica-Anthropos, 2012, 105 p.

A ndré Torre et Jean-Eudes Beuret proposent une contribution stimulante sur une problématique majeure, la question de la dynamique et de la gouvernance des territoires. Alors que cet ouvrage est de taille relativement modeste (105 p.), il est le fruit d'une contribution nous offrant une réflexion dense et motivante à partir du rôle des proximités. Les auteurs croisent une dimension scientifique et une dimension opérationnelle pour analyser les processus à l'œuvre dans la gouvernance des territoires. Il s'agit ainsi de réaliser un état des recherches mobilisant la notion de proximité comme fil conducteur de la réflexion, et d'y associer les derniers développements faisant appel aux modalités de coordination liées aux conventions, aux conflits et aux coopérations.

Torre et Beuret livrent un ouvrage clair et concis associant des notions théoriques et des exemples concrets issus de leurs pratiques, éclairant de façon originale les espaces ruraux et périurbains à travers les problématiques agricoles et environnementales. Par la rigueur de leur argumentation, ils contribuent à renouveler les cadres d'analyse et les outils mobilisés dans l'étude des dynamiques territoriales. Par leur construction à deux voix, ils nous proposent une réflexion intellectuelle rigoureuse mobilisant, non seulement une tradition de recherche solide, mais aussi étayée par de nombreux exemples issus de pratiques collectives diversifiées appliquées à ces espaces.

Cet ouvrage élabore un cheminement intellectuel à travers une architecture en six chapitres. Si les deux premiers ont pour vocation de poser le fondement de leur réflexion à travers l'explicitation du jeu des proximités géographiques et organisées dans la création et la dynamique des territoires, soubassement des processus de gouvernance, les chapitres suivants procèdent respectivement à une analyse approfondie des dimensions des processus de coordination, à savoir convention, conflit et concertation. À partir de la combinaison de ces trois modes de coordination, et plus particulièrement des conflits et de la concertation, naît alors la dynamique des territoires abordée dans le dernier chapitre. Ainsi, les auteurs font œuvre de pédagogie au fil des chapitres dont les notions sont toujours illustrées avec de très nombreux exemples appliqués.

Le premier chapitre présente les définitions élémentaires des proximités géographiques et organisées en soulignant par un rappel succinct les éléments fondamentaux et en montrant comment la proximité territoriale est issue de la combinaison des deux premières. Le chapitre deux questionne en quoi la gouvernance des territoires est produite par le jeu des proximités associant la dimension conflictuelle du processus de construction. Le chapitre trois aborde la dynamique des proximités comme moteur des processus de gouvernance territoriale. Le chapitre quatre mobilise la dimension de la concertation pour mettre en exergue la façon dont les acteurs utilisent la proximité géographique dans la construction de la proximité organisée. Le chapitre cinq s'interroge sur le rôle des conflits comme moteurs de la gouvernance. Enfin le chapitre six conclut l'ouvrage en proposant des formes d'action sur le jeu des proximités dans la dynamique des territoires.

L'originalité du propos est d'analyser la proximité territoriale comme le fruit des interactions entre proximités géographiques et organisées. Le processus d'émergence des proximités organisées qui s'élaborent sur le local, questionne 
l'exercice de la gouvernance et la possible fracture du territoire. Alors qu'en décembre 2012 nous quittait Albert Hirschman, les auteurs nous invitent à penser conjointement la concertation et le conflit. Bien qu'issus de processus distincts, la concertation et le conflit sont enchevêtrés pour stabiliser des équilibres temporaires, parfois instables, mais susceptibles d'apporter une pause dans la dynamique collective.

La montée attestée des conflits s'opère alors que les outils et leurs modalités de règlement se développent. Les auteurs soulignent deux dimensions singularisant les conflits d'usages de l'espace, le territoire et leur matérialité. Aussi ils associent ces conflits à la situation de Voice, ou prise de parole, leur conférant une dimension fédératrice pour les parties qui se recomposent lors de leur manifestation. Cette dynamique de la conflictualité leur permet de battre en brèche l'idée reçue selon laquelle le conflit serait uniquement négatif, oubliant sa capacité de fédération des acteurs dans la contestation. Ils montrent alors comment le conflit et la concertation fonctionnement ensemble pour impulser un processus dynamique. Cela les conduit à explorer les voies du comment penser la recomposition des proximités organisées à travers la concertation entre les acteurs. En quoi la combinaison entre les processus de construction des proximités géographiques temporaires et de la concertation peut-elle résoudre les problèmes posés par les proximités géographiques subies et par les recompositions des proximités organisées ? Cela revient donc à analyser les dynamiques de développement des territoires qui se fondent sur l'articulation conjointe entre conflits et concertations.

L'originalité de l'ouvrage est de proposer une décomposition fine du jeu complexe des oppositions et des coopérations que les acteurs locaux construisent dans l'élaboration du futur des territoires et dans leur gouvernance, dont la synthèse se lit dans la définition de projets et de trajectoires de développement territorial. Les auteurs nous offrent ainsi une grille d'analyse du jeu des proximités, proximités géographiques, subies ou recherchées, confrontées aux proximités organisées, d'appartenance ou de similitude, permettant de décrypter la gouvernance et les dynamiques de développement des territoires. Ce jeu dynamique souligne la façon dont les acteurs peuvent influencer la création de nouvelles proximités organisées, génératrices de dynamiques de coopération et de conflits.

Aussi les auteurs ouvrent-ils la réflexion à différents développements. Leurs travaux permettent, en effet, d'envisager de nouvelles combinaisons entre les proximités géographique et organisée, conduisant ainsi à réinterroger la création de sens comme moyen de réduire la distance entre les acteurs. Mais cette distance n'est-elle que d'essence géographique ? Les signes de qualité ont été introduits pour garantir le lien au lieu et pour permettre une certaine distanciation entre producteurs et consommateurs éloignés du lieu originel. Si ces signes ont permis, par la construction d'une proximité organisée, le développement d'une distanciation spatiale, ils procurent actuellement un réinvestissement du lien au lieu par une réappropriation locale. Affirmer la qualité de l'origine d'un produit s'enracine comme moyen de créer l'image et de valoriser le local, sources de nouveaux avantages compétitifs. Ces derniers ne sont pas uniquement marchands. Ils intègrent une dimension symbolique, vecteur d'identité et de recréation de sens. La réactivation du lien social dans une logique de similitude produit une spécificité, non plus seulement contenue dans le produit, mais également dans le processus qui l'ancre dans un espace géographique et culturel. Il s'agit dès lors de recréer du lien social entre les acteurs en local dans un mouvement plus global entremêlant conflits et concertations. Ces jeux réinventés entre les proximités réinterrogent la notion même de territoire, en soulignant 
sa complexité, sa mouvance et donc sa richesse.

Cet ouvrage est précieux. Comme ouvrage de réflexion pour la compréhension du jeu des proximités, et plus généralement pour l'analyse des processus de développement territorial, mais aussi comme outil d'aide à la décision et à l'action pour les acteurs publics et toute personne engagée ou non dans une action collective cherchant à penser, et à agir sur, la gouvernance des territoires.

Maryline FILIPPI Professeure Bordeaux Sciences Agro INRA SAD GAIA 